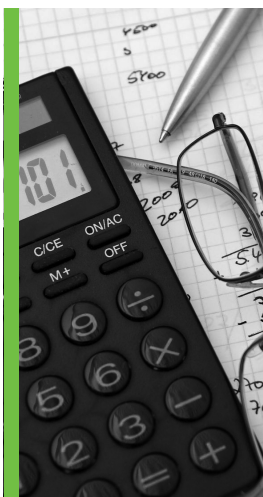

\title{
Beliebtheit und Akzeptanz von Phytopharmaka bei Publikum und Verordnern*
}

\author{
Rainer Stange
}

David Eisenberg konnte 1993 mit den Ergebnissen seiner ersten Befragung zur Praxis der Komplementärmedizin in den USA dem dortigen medizinischen Establishment in nahezu schockierender Weise vorführen, dass der Sektor „Complementary Medicine“ - gemessen an der Zahl der Patientenkontakte in der Allgemeinmedizin sowie am Geldeinsatz der Patienten - den damals in den USA üblichen Privatzahlungen für Krankenhausaufenthalte gleichkam (1). Darüber hinaus konnte er durch wiederholte Anwendung desselben Erhebungsinstrumentes 7 Jahre später für diesen Tatbestand einen historisch positiven Trend nachweisen (2). Seine beiden diesbezüglichen Veröffentlichungen in renommierten Zeitschriften trugen ganz entscheidend zur nachfolgend zunehmenden Anerkennung der Komplementärmedizin in der medizinischen Praxis, der Ausbildung und nicht zuletzt der großzügig durch die Regierung unterstützten Forschung in den USA bei.

Eine vergleichbar griffige Einschätzung der unmittelbaren therapeutischen wie öko- nomischen Bedeutung von Naturheilverfahren bzw. Komplementärmedizin oder einer Komponente wie der Phytotherapie ist für hiesige Verhältnisse nicht bekannt geworden.

Die Bedeutung der Phytotherapie in Deutschland kann jedoch ansatzweise mit mehreren Untersuchungsinstrumenten erfasst und z.T. auch in ihrer Entwicklung beobachtet werden. Dabei werden durch verschiedene Messinstrumente zwangsläufig unterschiedliche Fragestellungen beantwortet. Es bieten sich insbesondere an:

$>$ stochastische Umfragen in der Bevölkerung (unterschiedslos Gesunde wie Patienten)

$>$ Umfragen unter Zielgruppen, insbesondere:

- Patienten mit einer gesichert diagnostizierten Erkrankung - professionelle Akteure, insbesondere Ärzte, Apotheker, Heilpraktiker

$>$ Daten der Kostenträger

$>$ Marktdaten

\section{ZUSAMMENFASSUNG}

Die Phytotherapie wird in der medizinischen Versorgung insgesamt, aber auch innerhalb von Naturheilverfahren und der Komplementärmedizin als sehr bedeutsam erlebt. Hier wird versucht, ihren Stellenwert mit verschiedenen Messinstrumenten zu erfassen. Danach weisen mehrere Untersuchungen eine ausreichende Übereinstimmung der ausgeprägten Inanspruchnahme durch die Bevölkerung nach. Dagegen erfasst nur eine das Verordnungsverhalten und zeigt die deutlich höhere Beliebtheit dieser Heilverfahren bei Primär- als bei Fachärzten. Marktdaten können Trends noch zuverlässiger als die Meinungsforschung beschreiben.

\section{Schlüsselwörter}

Phytotherapie, Umfragen, 12-Monatsprävalenz, Verordnungsverhalten, OTC-Markt

\section{Bevölkerungsumfragen}

Wissenschaftliche Untersuchungen sind insbesondere aus repräsentativen Bevölkerungsumfragen veröffentlicht worden. I.d.R. wird die 12-Monatsprävalenz der Inanspruchnahme einer bestimmten Therapie, manchmal auch eines Spektrums wie „Naturheilverfahren“ oder „Komplementärmedizin“ abgefragt. Insofern können Ergebnisse aus ganz unterschiedlichen Quellen verglichen werden, z.B.:

$>$ Gesellschaft für Konsumforschung

> Bertelsmann Gesundheitsmonitor Bevölkerung

$>$ Sebastian-Kneipp-Institut

$>$ Institut für Demoskopie Allensbach

Die Gesellschaft für Konsumforschung Nürnberg (GfK) führte im Mai 2007 unter 1978 stochastisch ermittelten über 14-jährigen Bundesbürgern eine Befragung durch, die neben der 12-Monatsprävalenz der persönlichen Inanspruchnahme von 10 Gebieten bzw. Methoden der Naturheilverfahren und Komplementärmedizin auch dichotomisiert (erfolgreich - nicht erfolgreich) die persönliche Erfolgsbeurteilung der jeweiligen Therapie erhob (3). Die Prävalenz für die Phytotherapie war mit 33,4\% die höchste, mit deutlichem Abstand gefolgt von Akupunktur mit 17,8\% (Abb. 1). Die Erfolgsquote von $68 \%$ war mit den 4 weiteren am häufigsten genutzten Verfahren vergleichbar.

Vergleichszahlen zur Inanspruchnahme liefert der „Bertelsmann Gesundheitsmo-

\footnotetext{
* Nach einem Vortrag des Autors beim „Expertenmeeting zur Phytotherapie“ (Veranstalter: Charité und Immanuel Krankenhaus Berlin) am 6.12.2013 in Berlin.
} 
nitor Bevölkerung“ (Abb. 2). Er stellt zu verschiedenen Zeitpunkten jeweils verschiedenen Personen dieselben Fragen zu Gesundheitsbewusstsein und -versorgung. Da die Stichproben nach denselben Kriterien aus Bevölkerungsregistern gezogen werden, lassen sich aus den Daten verlässlich Trends ableiten. In der Beurteilung des Zeitverlaufs fällt zwischen 2005 und 2006 ein dramatischer Rückgang der über alle Verfahren summierten 12-Monatsprävalenz von 57 auf $43 \%$ auf, der sich abgeschwächt auf $40 \%$ bis 2012 fortsetzte (Abb. 3), als 63\% der Befragten eine Lebenszeitprävalenz (Inanspruchnahme ohne zeitliche Begrenzung) aufwiesen. $31 \%$ der Verwender zählten zu sog. Vielnutzern, d.h. sie hatten lebenszeitlich bereits 3 oder mehr unterschiedliche Verfahren in Anspruch genommen (4).

Die Autoren führen dies vor allem auf einen Einbruch in der Verwendung von Naturheilmitteln und pflanzlichen Arzneien zurück. Sehr wahrscheinlich sei dieser Effekt eine direkte Nachwirkung der seit Anfang 2004 geltenden Änderung des § 34a Sozialgesetzbuch $\mathrm{V}$, nach der frei verkäufliche Arzneimittel nur noch nach in sehr präzise und sparsam erlassenen Ausnahmeregelungen von der gesetzlichen Krankenversicherung (GKV) erstattet werden. Dies ist in der zeitlichen Darstellung der Ergebnisse gut nachvollziehbar, während andere Therapierichtungen, die von dieser Änderung weniger oder gar nicht betroffen waren, einen solchen Einbruch nicht erfahren haben. Interessant sei jedoch, dass sich dieser Einbruch in den Allensbach-Umfragen zum Thema Naturheilmittel, die ebenfalls einen kleinen Satz von Fragen regelmäßig stellen (5), bisher nicht gezeigt hätte (4). Diese Umfrage erhob auch ein subjektives Erfolgskriterium. Hier antworteten für die „Naturheilmittel“, d.h. im wesentlichen Phytopharmaka, $46 \%$,ja, hat gut geholfen“ und $40 \%$,ja, hat etwas geholfen“. Dieses summierte Kriterium wird lediglich von Chirotherapie/Osteopathie mit $89 \%$ übertroffen.

In einer repräsentativen Bevölkerungsumfrage des ehemaligen Europäischen Gesundheitszentrums für Naturheilverfahren, Sebastian-Kneipp-Institut GmbH Bad Wörishofen erhoben geschulte Telefonin-

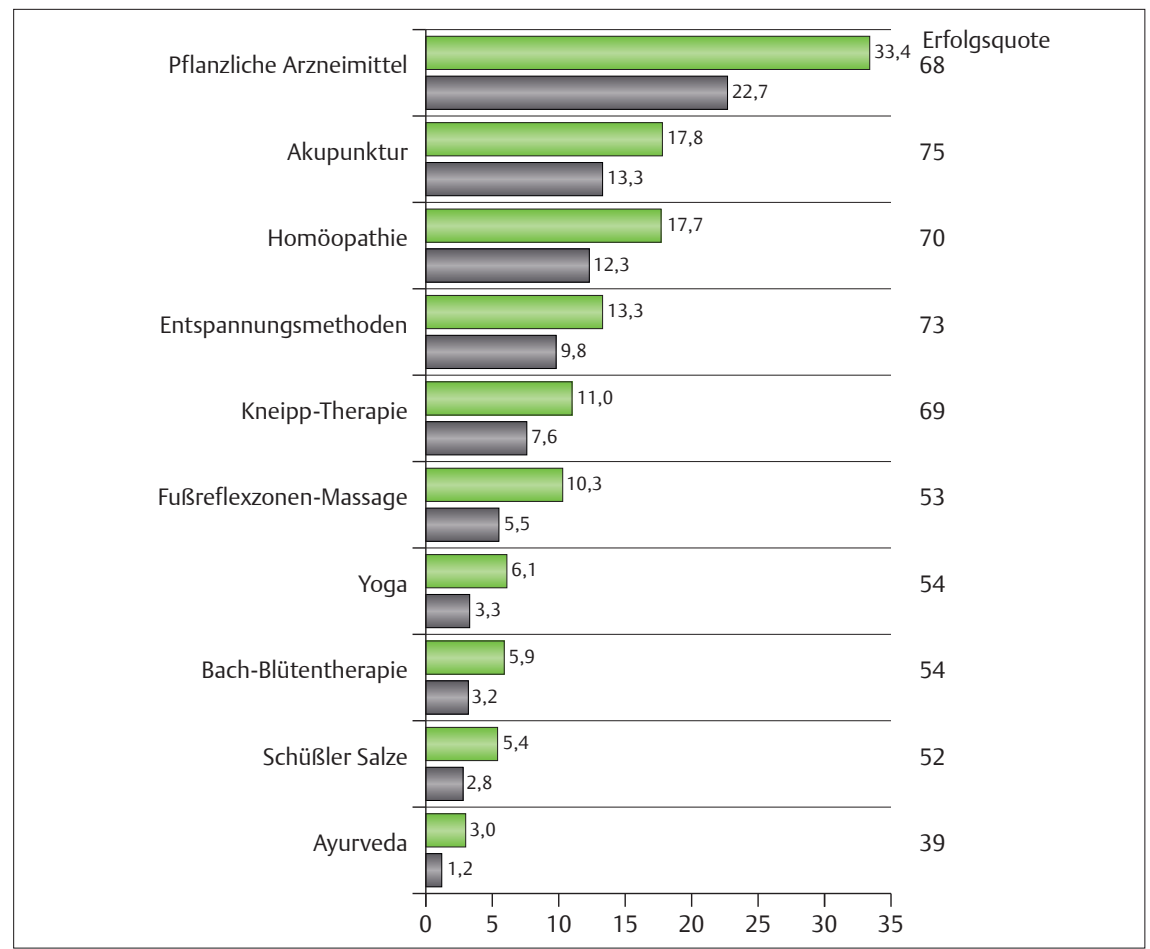

Abb.1: 12-Monatsprävalenzen nach Therapieverfahren (oberer Balken) mit Selbstbeurteilung des Erfolges (ja/nein) (unterer Balken); n= 1978. GfK 2007.

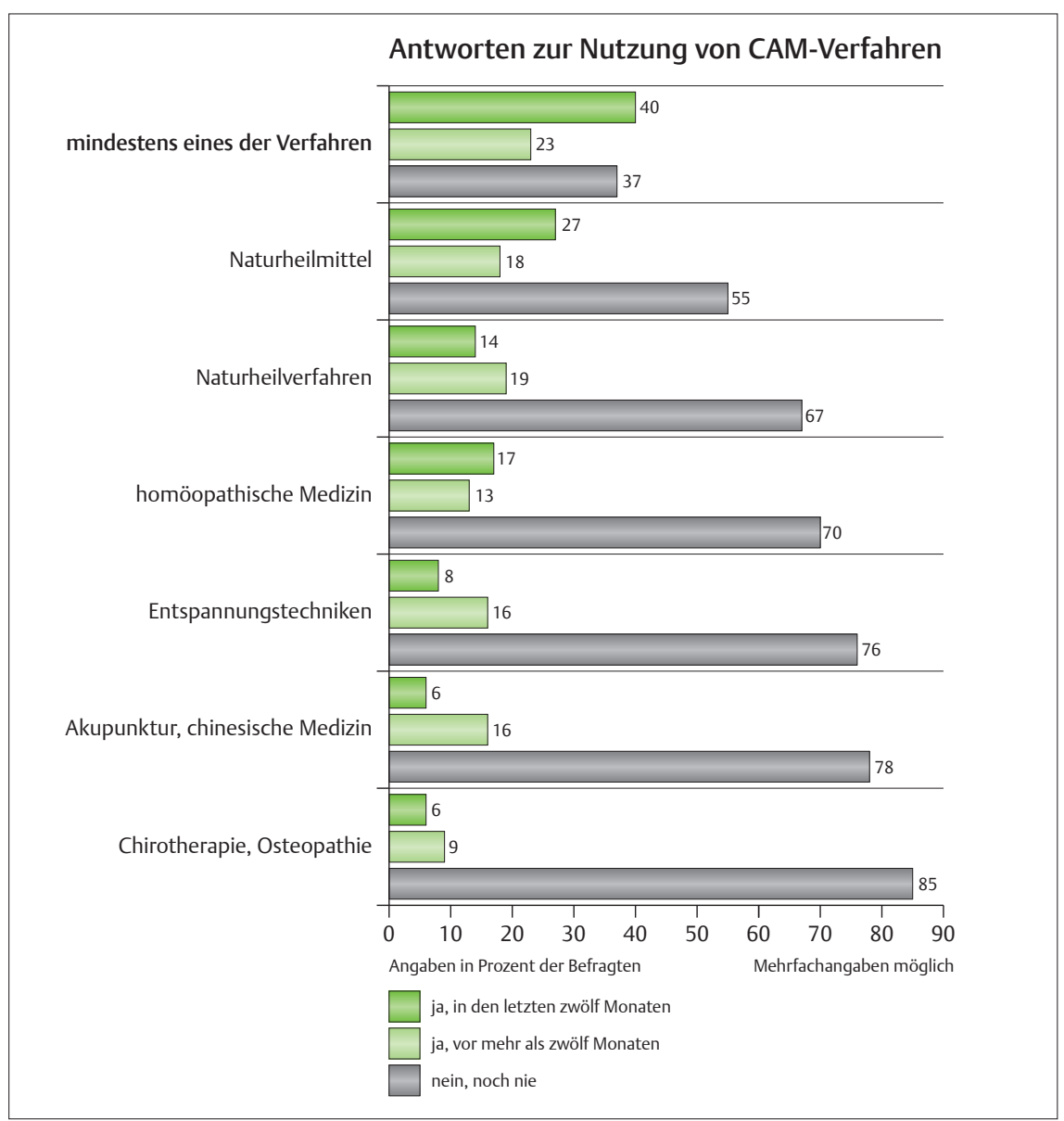

Abb.2: Gesundheitsmonitor der Bertelsmann Stiftung, Bevölkerungsumfrage 2012 (4). 


\section{Anwendung der einzelnen Verfahren in den vergangenen zwölf Monaten im Vergleich der unterschiedlichen Befragungswellen}

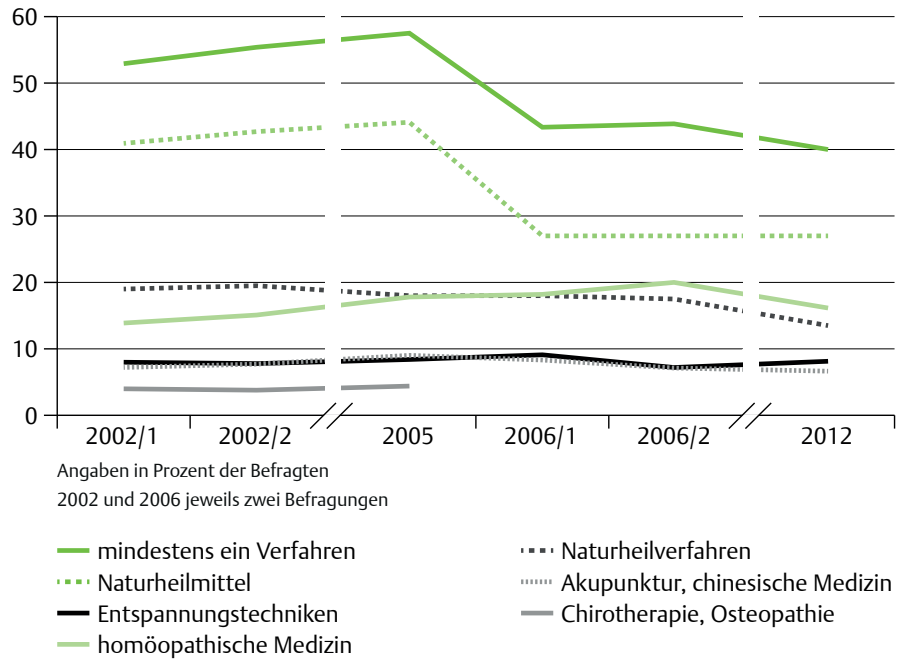

Abb. 3: Gesundheitsmonitor der Bertelsmann Stiftung, Längsschnitt der Bevölkerungsumfragen (4).

terviewer bei 1100 Befragten im Jahr 2002 eine 12-Monatsprävalenz für alle Verfahren von $62,3 \%$ (70,9\% bei Frauen, 53,9\% bei Männern) (Tab. 1) (6). Für die „Heilpflanzentherapie“ ergaben sich 26,6\% (Frauen 32,7\%, Männer 20,4\%). Dieser Begriff war statt Phytotherapie in der Originalfrage gewählt worden und wurde bei Nachfrage expliziert, insbesondere um dem Befragten eine sichere Abgrenzung zur ebenfalls abgefragten Homöopathie zu ermöglichen. Auch hier fällt auf, dass die Phytotherapie unmittelbar nach der Bewegungstherapie, die die Gesellschaft für Konsumforschung nicht abgefragt hatte, eine führende Position innehat. Der etwas geringere Anteil von 26,6\% (Kneipp) vs. 33,4\% (GfK) könnte in der etwas schärferen Beurteilung durch die Interviewer begründet liegen.

Die Daten der Umfrage des SebastianKneipp-Instituts lassen sich auch mit denen des „Bertelsmann Gesundheitsmonitors Bevölkerung“ vergleichen (s.o. Abb. 2). In Letzterem wurde explizit nach „Naturheilmitteln“ gefragt. Darunter versteht man v.a. die Phytotherapie, aber auch die mikrobiologische Therapie sowie tierisch abgeleitete Heilmittel (z.B. bestimmte Enzyme) lassen sich einschließen, dürften jedoch nur eine untergeordnete Rolle spielen. Als wichtigstes und erstaunlich gut übereinstimmendes Ergebnis ergeben sich für die 12-Monatsprävalenz jeweils etwa $27 \%$.

\section{Patientenumfragen}

Patienten weisen je nach ihrer Erkrankung davon abweichende und i.d.R. eher spezifische, d.h. auf bestimmte Pflanzen fokussierte Interessen für Phytotherapie auf. Als Beispiel kann die bundesweite Frühkohortenstudie des Deutschen Rheumaforschungszentrums Berlin von 2005 dienen, in der 916 Patienten bis maximal 3 Jahre nach Erstdiagnose einer chronischen Polyarthritis nach der Inanspruchnahme von Naturheilverfah- ren und Komplementärmedizin gefragt wurden. Da hier in erster Linie die Prävalenz seit Diagnosestellung interessierte, wurde wegen der z.T. weniger als 12 Monate zurückliegenden Erstdiagnose die 6-Monatsprävalenz abgefragt (7). 42\% der Patienten hatten mindestens eine der 32 vorgeschlagenen Maßnahmen angewandt, etwa die Hälfte von ihnen mehrere. Eine Vorerfahrung mit „Anti-inflammatorisch wirksamen Phytotherapeutika“ gaben $18,2 \%$ an, darunter $10,8 \%$ mit der damals für die Phytotherapie als führend erachteten und prioritär abgefragten Nutzung von Teufelskralle. Nicht erfragt wurden Änderungen von Einstellungen und Praxis infolge der Erkrankung sowie eine Wirksamkeitsbewertung.

\section{Einstellung und Verordnungsge- wohnheiten von Ärzten}

Diese wurden bislang nur selten untersucht. Der Bertelsmann Gesundheitsmonitor hat diese in einer Umfrage unter niedergelassenen Ärzten mit GKV-Zulassung für das Jahr 2005 sehr detailliert erfasst. Danach gaben über alle Gruppen niedergelassener Ärzte $28 \%$ an, sehr häufig sowie weitere $39 \%$ gelegentlich Phytopharmaka zu verordnen (Abb. 4) (8).

Wenn das Ergebnis nach Fachgruppen analysiert wurde, ergaben sich ähnliche Unter-

Tab. 1: 12-Monatsprävalenzen nach Therapieverfahren und Geschlecht. Sebastian-Kneipp-Institut 2002 (6).

\begin{tabular}{|c|c|c|c|}
\hline & $\begin{array}{l}\text { gesamt } \\
(n=1100)\end{array}$ & $\begin{array}{l}\text { Frauen } \\
(n=549)\end{array}$ & $\begin{array}{l}\text { Männer } \\
(\mathrm{n}=551)\end{array}$ \\
\hline $\begin{array}{l}\text { Anwendung mind. eines Naturheilverfahrens oder } \\
\text { einer eigenständigen medizinischen Richtung }\end{array}$ & 62,3 & 70,9 & 53,9 \\
\hline \multicolumn{4}{|l|}{ Klassische NHV } \\
\hline Bewegungstherapie & 29,3 & 32,2 & 26,4 \\
\hline Heilpflanzentherapie & 26,6 & 32,7 & 20,4 \\
\hline Hydrotherapie & 20,6 & 24,3 & 16,9 \\
\hline medizinische Massagen & 18,2 & 22,0 & 14,5 \\
\hline Thermotherapie & 14,5 & 17,9 & 11,2 \\
\hline Ernährungstherapie & 13,0 & 14,0 & 11,9 \\
\hline Balneotherapie & 7,8 & 9,9 & 5,7 \\
\hline Ordnungstherapie & 4,7 & 5,4 & 3,9 \\
\hline $\begin{array}{l}\text { Anwendung mind. eines klassischen Naturheil- } \\
\text { verfahrens in den letzten } 12 \text { Monaten }\end{array}$ & 57,0 & 64,6 & 49,5 \\
\hline
\end{tabular}




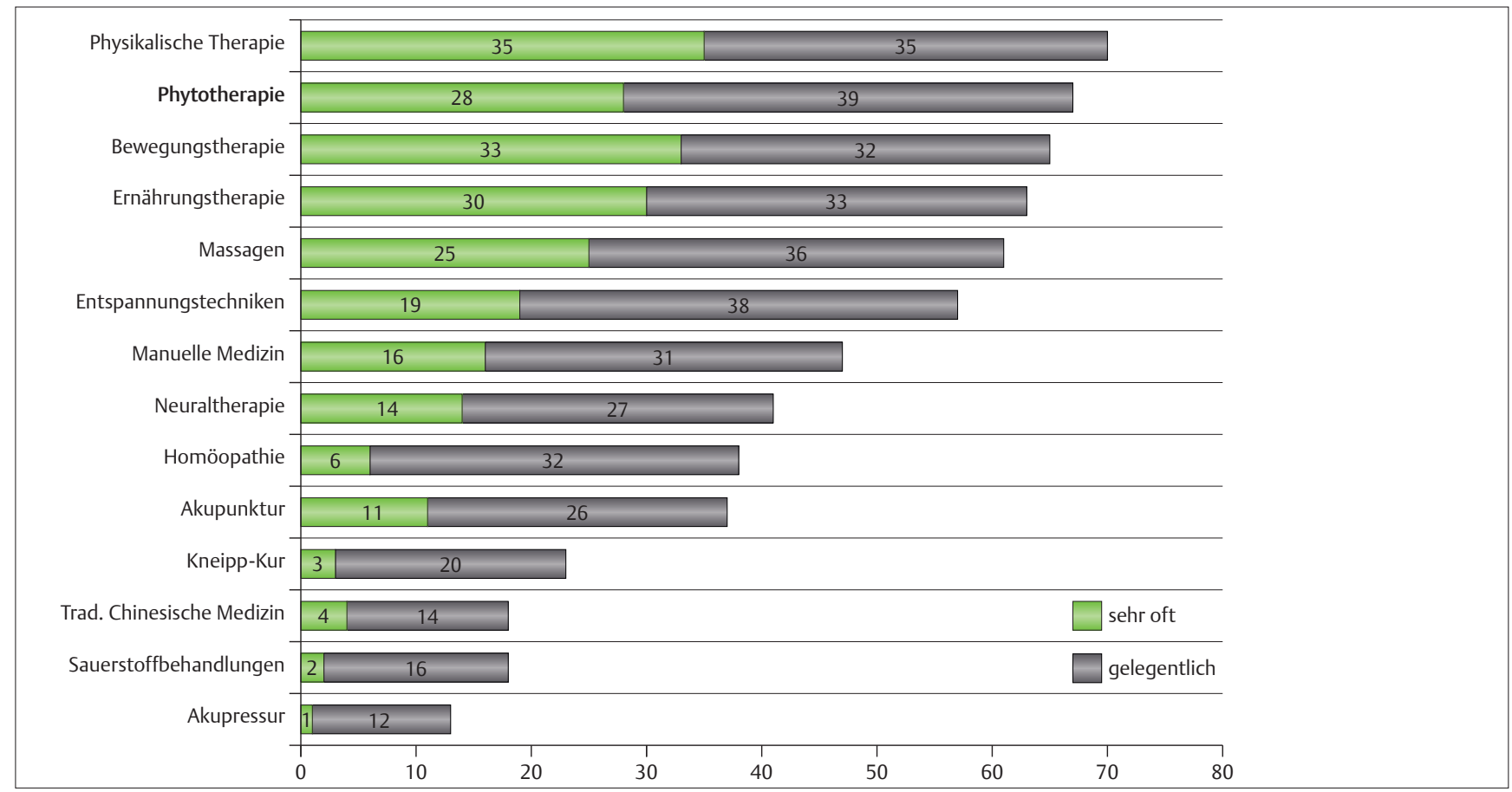

Abb.4: 12-Monatsprävalenz der Verordnung „alternativer Heilmethoden“ (in \% von 512 deutschen Kassenärzten, summiert Primärärzte und Fachärzte) (8).

schiede wie für die anderen abgefragten Verfahren aus Naturheilkunde und Komplementärmedizin. Sog. Primärärzte, hier verstanden als Hausärzte, Gynäkologen und Pädiater, hatten zu $88 \%$ in den vergangenen 12 Monaten Phytopharmaka verordnet, Fachärzte gemittelt über alle Richtungen dagegen nur zu 54\% (Abb. 5).

\section{Ausgaben der Kostenträger}

Die Ausgaben der GKV-Kostenträger werden für ausgewählte Indikationsgruppen sehr exakt z.B. durch das Institut für Gesundheitssystemforschung Berlin (IGES) errechnet und publiziert. Daraus ergibt sich für die Jahre 2004 und 2005 etwa für das Indikationsgebiet Urologika ein deutlicher Rückgang, der angedeutet auch für Antiphlogistika und Antirheumatika nachzuvollziehen ist (Abb. 6, Abb. 7). Für beide Gruppen dürfte dies in der oben beschriebenen Gesetzgebung begründet liegen. Das unterschiedliche Ausmaß des Einbruchs erklärt sich durch die Anteile der Phytotherapeutika in den Gruppen - bei den Urologika etwa waren zuvor $\beta$-sitosterinhaltige Phytopharmaka in der Therapie der Prostatahyperplasie sehr verbreitet, während Phytoanalgetika gegenüber z.B. NSAR nur eine untergeordnete Rolle spielen.
Weitere Marktdaten ergeben sich aus Forschungsberichten wie dem IMS OTC-Report oder der IMS Gesundheitsmittelstudie. Der OTC-Markt wird sehr wesentlich von Phytopharmaka bestimmt. Auch hier

spiegeln sich die Entwicklungen der Gesundheitsreform 2004 insofern wider, als die Relation zwischen GKV-Kostenübernahme und Selbstmedikation konstant erniedrigt bleibt (Abb. 8). Die PKV ist dage-

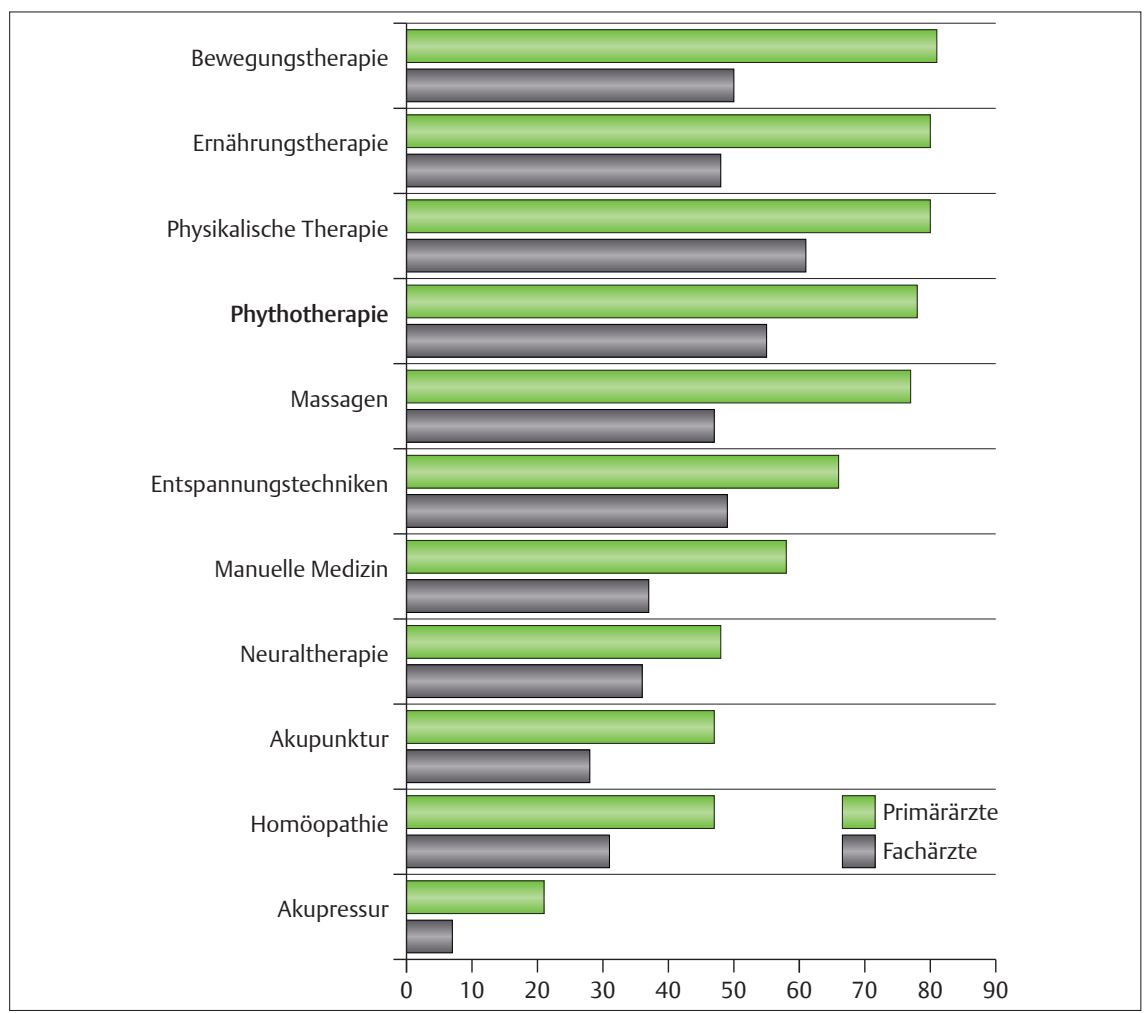

Abb.5: 12-Monatsprävalenz der Verordnung „alternativer Heilmethoden“ (in \% von 512 deutschen Kassenärzten, differenziert nach Primärärzten und Fachärzten) (8). 


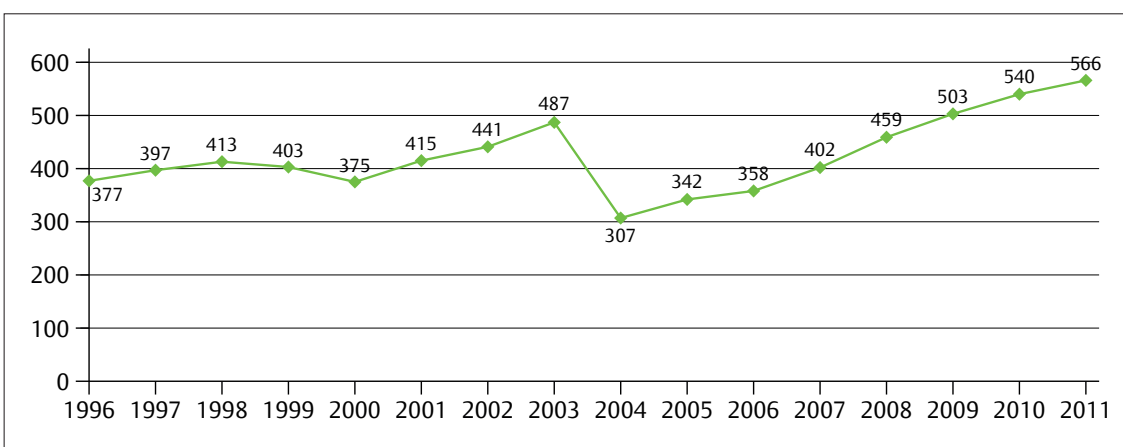

Abb.6: Verbrauch an Urologika in der GKV in Mio. DDD 1996-2011 (aus IGES-Arzneimittelatlas 2012) (9).

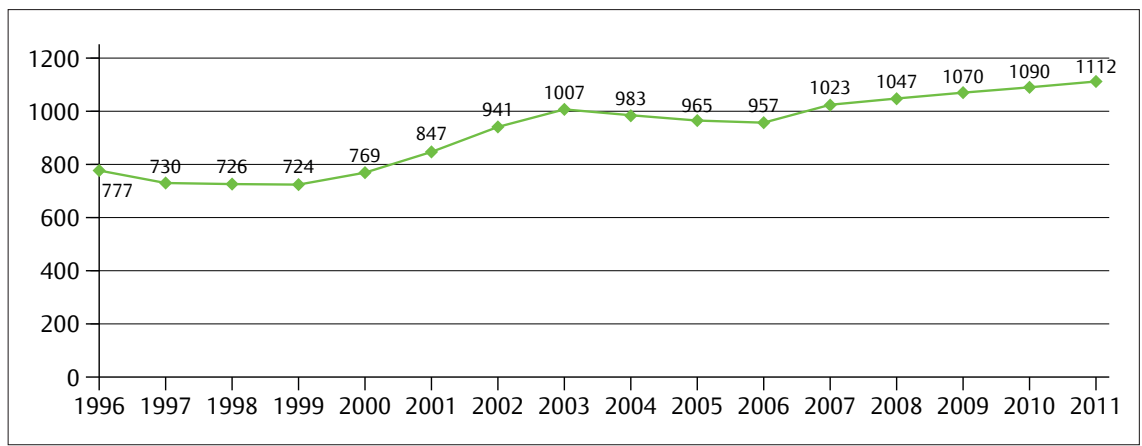

Abb.7: Verbrauch an Antiphlogistika und Antirheumatika in der GKV in Mio. DDD 1996-2011 (aus IGES-Arzneimittelatlas 2012) (9).

gen - verglichen mit ihren Anteil an Versicherten - deutlich überrepräsentiert. Insgesamt ergeben sich für den OTC-Markt jetzt wieder schwache Wachstumstendenzen, die bezüglich der Verkaufswege den Versandhandel begünstigen (Abb. 9).

\section{Zusammenfassung und Ausblick}

In 2 repräsentativen Bevölkerungsumfragen ergab sich die 12-Monatsprävalenz für die Inanspruchnahme von „Naturheilmit-

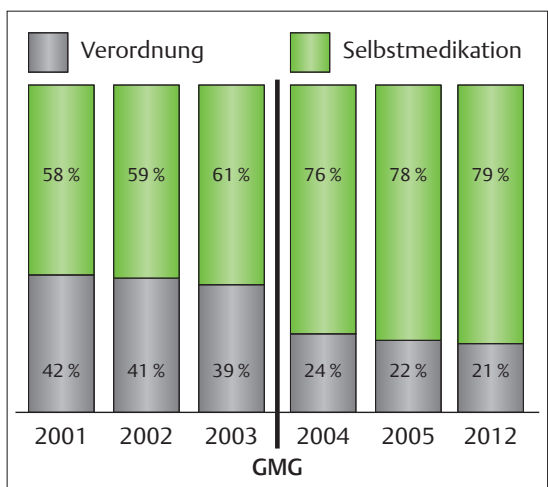

Abb. 8: Relative Kostenaufteilung für den gesamten OTC-Umsatz in Deutschland zwischen GKV und Selbstzahlern, Balkenhöhe entspricht 100\%; gibt nicht die absoluten Beträge wieder (10). teln“ bzw. „Phytotherapie“ übereinstimmend zu $27 \%$. In den 2 bekannt gewordenen Umfragen mit Selbsteinschätzung des Therapieerfolgs wird dieser mit 68\% (GfK, 1 Kriterium) bzw. 86\% (Bertelsmann, 2 Kriterien) angegeben.

Rheumapatienten (RA) mit hoher Affinität zu einer leitliniengerechten Therapie haben im 3. Jahr nach Erstdiagnose krankheitsspezifische Erfahrungen zu 19,4\% für alle Phytotherapeutika und zu 10,8\% für Teufelskralle als meistgenannte einzelne Pflanze.

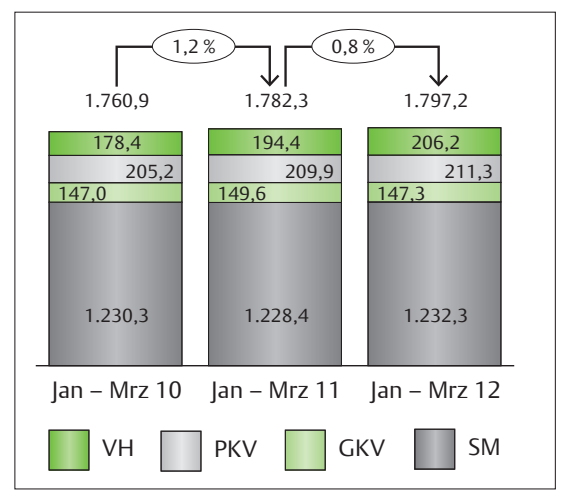

Abb. 9: OTC-Markt in Deutschland jeweils JanuarMärz 2010-2012 in Mio. € zu Apothekenverkaufspreisen, aufgeteilt nach Versandhandel (VH), PKV, GKV, Selbstmedikation (SM) (11).
Unter Ärzten aller Fachgruppen verordnen $28 \%$ „sehr oft“ bzw. 39\% „gelegentlich“ Phytopharmaka, Fachärzte jedoch seltener als Primärärzte.

Umfragen wie Markdaten lassen zumindest für ausgewählte Indikationsgruppen den deutlichen Einbruch nach dem GMG 2004 erkennen, der nicht wieder kompensiert werden konnte. Im gesamten OTCMarkt scheint dagegen in den letzten Jahren eine Stabilität eingetreten zu sein, zu der ein sehr hoher Anteil Selbstzahler sowie deutlich überproportional die PKV als Kostenträger beitragen.

Die angeführten Untersuchungen scheinen ausreichend geeignet, die Inanspruchnahme der Phytotherapie in Deutschland zu quantifizieren. Weitere Untersuchungen sollten sich auf deren Erfolge aus Patienten- wie Arztsicht sowie pharmaökonomische Auswirkungen unter Alltagsbedingungen konzentrieren.

Dr. med. Rainer Stange

Abteilung Naturheilkunde

Immanuel Krankenhaus Berlin-Wannsee

Königstr. 63

14109 Berlin

r.stange@immanuel.de

- Online

http://dx.doi.org/10.1055/s-0033-1349783

\section{LITERATUR}

1 Eisenberg DM, Kessler RC, Foster C et al. Unconventional medicine in the United States. Prevalence, costs, and patterns of use. N Engl J Med 1993; 328: 246-252

2 Eisenberg DM, Davis RB, Ettner SL et al. Trends in alternative medicine use in the United States, 1990-1997: results of a follow-up national survey. JAMA 1998; 280: 1569-1575

3 Apotheken Umschau 2009, Heft 13, S. 11

4 Linde K, Buitkamp M, Schneider A, Joos J. Naturheilverfahren, komplementäre und alternative Therapien. In: Böcken J, Braun B, Repschläger U, Hrsg. Gesundheitsmonitor 2012. Gütersloh: Verlag Bertelsmann Stiftung; 2013: 118-135 
5 Institut für Demoskopie Allensbach. Naturheilmittel 2010 - Ergebnisse einer bevölkerungsrepräsentativen Befragung.

http://www.ifd-allensbach.de/uploads/

tx_studies/7528_Naturheilmittel_2010.pdf (Download 10.7.2012)

6 Härtel U, Volger E. Inanspruchnahme und Akzeptanz klassischer Naturheilverfahren und alternativer Heilmethoden in Deutschland - Ergebnisse einer repräsentativen Bevölkerungsstudie. Forsch Komplementärmed Klass Naturheilkd 2004; 11: 327-334

7 Westhoff G, Stange R, Zink A. Patients with recent onset rheumatoid arthritis use complementary and alternative medicine irrespective of disease activity and kind of care. Forsch Komplementärmed 2007; 14 (Suppl. 1): 47

8 Stange R, Amhof R, Moebus S. Naturheilverfahren, Komplementär- und Alternativmedizin im Bewußtsein und Handeln von niedergelassenen Ärzten. In: Böcken J, Braun B, Amhof R, Schnee M, Hrsg. Gesundheitsmonitor 2006. Gütersloh: Verlag Bertelsmann Stiftung; 2006: 208-232
9 IGES Institut für Gesundheitssystemforschung Berlin. Arzneimittelatlas 2012. Zitiert aus http://www.iges.de (Download 5.12.2013)

10 Quelle: http://www. healthcaremarketing.eu (Download 4.12. 2013)
11 IMS, Hrsg. IMS Marktbericht: Entwicklung des deutschen Pharmamarktes im März 2012, S. 19. http://www.imshealth.com/ ims/Global/EMEA/Austria\%20and\% 20Germany/Home\%20Page\%20Content/ IMS\%20News/2012_03_IMS\%20Marktbericht.pdf (Download 4.12.2013)

\section{SUMMARY}

\section{Popularity, acceptance and regulation of phytotherapy}

Phytotherapy plays a very significant role in medical care as a whole and thus, also in complementary and alternative medicine. This article seeks to capture the importance of phytotherapy by using various measuring instruments. Accordingly, several surveys have demonstrated a sufficient harmony in the application of phytotherapeutic remedies by the population. However, only one nationwide survey deals with the practice of prescription and shows the significantly higher popularity of phytotherapy in primary care than in specialized medical practice. The author of this article suggests that marketing data can be used to describe trends more reliably than demoscopic research.

\section{Key words}

Phytotherapy, public opinion survey, 12-months prevalence, OTC-market 